\title{
Outcome of Stroke Patients with Cancer and Nonbacterial Thrombotic Endocarditis
}

\author{
Joonsang Yoo, ${ }^{\mathrm{a}, \mathrm{b}}$ Jin Kyo Choi, ${ }^{\mathrm{b}}$ Young Dae Kim, ${ }^{\mathrm{b}, \mathrm{c}}$ Hyo Suk Nam, ${ }^{\mathrm{b}, \mathrm{c}}$ Hyungjong Park, ${ }^{\mathrm{b}, \mathrm{d}}$ Hye Sun Lee, \\ Ji Hoe Heo ${ }^{\text {b,c }}$

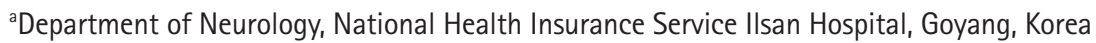 \\ ${ }^{b}$ Department of Neurology, Yonsei University College of Medicine, Seoul, Korea \\ 'Integrative Research Center for Cerebrovascular and Cardiovascular Diseases, Yonsei University College of Medicine, Seoul, Korea \\ 'Department of Neurology, Keimyung University School of Medicine, Daegu, Korea \\ 'Biostatistics Collaboration Unit, Yonsei University College of Medicine, Seoul, Korea
}

Background and Purpose Nonbacterial thrombotic endocarditis (NBTE) is a cause of stroke in cancer. However, clinical characteristics and outcomes in stroke patients with cancer-associated NBTE are not well known.

Methods We included consecutive patients with stroke and active cancer over a 9-year period who underwent echocardiography. We retrospectively compared clinical characteristics and presence of metastasis between patients with NBTE, those with cryptogenic etiologies, and those with determined etiologies. We also investigated mortality and stroke events during the 6-month follow-up.

Results Among the 245 patients, 20 had NBTE, 96 had cryptogenic etiologies, and 129 had determined etiologies. Metastasis was seen in all 20 patients (100\%) with NBTE, 69.8\% in patients with cryptogenic etiology, and $48.8 \%$ in patients with or determined etiology. During the 6-month follow-up, 127 patients (51.8\%) developed stroke and/or died (death in 110 [44.9\%] and stroke events in 55 [22.4\%]). Patients with NBTE showed significantly higher mortality (80\%) and stroke occurrence (50\%) than those with cryptogenic etiologies (mortality 54.2\%, stroke 25.0\%, log-rank $P=0.006$ ) and determined etiologies (mortality $32.6 \%$, stroke $16.3 \%$, log-rank $P<0.001$ ). In a multivariate Cox proportional hazard analysis, the presence of NBTE was independently associated with composite outcomes of mortality and stroke events (hazard ratio, 1.941; 95\% confidence interval, 1.052 to 3.690$)$.

Conclusions NBTE should be suspected as a potential cause of stroke in patients with metastatic cancer. Patients with NBTE have a high risk of recurrent stroke and mortality. Future studies are necessary to determine strategies to reduce stroke recurrence in patients with NBTE.
Correspondence: Ji Hoe Heo Department of Neurology, Yonsei University College of Medicine, 50-1 Yonsei-ro, Seodaemun-gu, Seoul 03722 , Korea

Tel: $+82-2-2228-1605$

Fax: +82-2-393-0705

E-mail:jhheo@yuhs.ac

Received: February 26, 2020

Revised: April 20, 2020

Accepted: April 21, 2020

Keywords Stroke; Neoplasms; Metastasis; Mortality; Nonbacterial thrombotic endocarditis

\section{Introduction}

Cancer may be associated with stroke. In a large cohort study, the 6-month cumulative incidence of ischemic stroke was sig- nificantly higher in patients with cancer than in matched controls (3.0\% vs. $1.62 \%) .{ }^{1}$ Nonbacterial thrombotic endocarditis (NBTE) is characterized by noninfectious vegetation on the cardiac valves. ${ }^{2}$ NBTE is a cause of stroke and most frequently 
develops in patients with systemic cancer. ${ }^{3,4}$

Most information on NBTE in stroke is derived from case reports or post-mortem studies because of its rarity. ${ }^{5-8}$ Although patients with acute stroke and active cancer are known to have a substantial short-term risk of recurrent stroke, ${ }^{9}$ the outcomes of stroke patients with cancer-associated NBTE are not well known. In addition, the pathomechanism of NBTE is unknown. However, pathological studies have shown that the vegetation and thrombi retrieved from stroke patients with NBTE have very high platelet fractions. ${ }^{10,11}$ Further, cancer is known to activate platelets, which play an important role in metastasis. ${ }^{12}$ These findings suggest a potential association between NBTE and metastasis. Previous case reports and post-mortem studies have shown frequent occurrences of metastasis in patients with NBTE. However, information on the presence of metastasis is insufficient in many cases. ${ }^{13-16}$ Therefore, the association between NBTE and metastasis remains uncertain.

We hypothesized that development of NBTE has a relationship with metastasis. In this study, we investigated association between NBTE and metastasis, and the mortality and occurrence of stroke events for 6 months following an initial stroke in patients with cancer-associated NBTE.

\section{Methods}

\section{Study population}

This retrospective study used a hospital-based prospective cohort of consecutively enrolled patients with ischemic stroke within 7 days of symptoms onset. Routine evaluations performed were as follows: standard blood tests including D-dimer; brain magnetic resonance imaging (MRI) and/or computed tomography (CT) and cerebral angiography (magnetic resonance angiography, CT angiography, or conventional angiography); Holter monitoring or continuous electrocardiography monitoring during admission in the stroke unit; and transesophageal echocardiography (TEE) and/or transthoracic echocardiography (TTE). TEE was not performed in patients with decreased consciousness, aphasia, impending brain herniation, poor systemic conditions, severe swallowing difficulty, tracheal intubation, or when informed consent could not be obtained. ${ }^{17}$

The study hospital is a tertiary university hospital with a dedicated cancer center where patients with cancer routinely undergo extensive evaluations for the presence of metastasis. When cancer or NBTE is diagnosed during admission, patients are referred to oncologists for further evaluation and treatment.

This study included consecutive patients with active cancer who were registered between January 2010 and December 2018 and who underwent echocardiography. Active cancer was defined as cancer that was diagnosed within 6 months, required chemotherapy or surgical treatment within 6 months, or was recurrent, metastatic, or inoperable. ${ }^{18}$ Patients with brain malignancy or hematologic malignancy were excluded. The cohort and this study were approved by the Institutional Review Board of the Yonsei University Health System. The need for an informed consent was waived due to the retrospective nature of the study.

\section{Study group}

Because the study participants had active cancer, patients were categorized into those with NBTE, those with cryptogenic etiology, and those with determined etiology. ${ }^{11}$ NBTE was determined based on formal reports by cardiologists who diagnosed vegetation based on echocardiographic examinations. Five patients with NBTE and co-existing atrial fibrillation were classified into the NBTE group. Twenty-one patients with a patent foramen ovale without venous thrombosis and other determinable etiologies were classified into cryptogenic etiology group.

\section{Outcome assessments}

All patients were regularly followed up after discharge by neurologists and clinical research assistants in the outpatient clinic using face-to-face interviews or by a telephonic interview using a structured questionnaire. ${ }^{19}$ We reviewed the medical records to obtain information on mortality and cause of death. The cause of death was categorized as stroke-related, cancerrelated, or others/unknown. ${ }^{20}$ Information on the occurrence of any new stroke events (cerebral infarction or intracerebral hemorrhage $[\mathrm{ICH}])$ after the initial cerebral infarction was also obtained from medical records. The diagnosis of stroke was based on brain $\mathrm{MRI}$ and/or $\mathrm{CT}$ results.

\section{Statistical analysis}

Variables were expressed as mean \pm standard deviation, median (interquartile range), or number (percentage), as appropriate. Baseline characteristics among the groups were compared using one-way analysis of variance or the Kruskal-Wallis test for continuous variables and the chi-square test or Fisher's exact test for categorical variables. Post hoc analysis was performed using Bonferroni's correction or Dunn's test. Kaplan-Meier estimates and log-rank tests were performed (adjusted by Bonferroni's correction) for mortality, occurrence of stroke, and composite outcomes of mortality and stroke. To determine whether the presence of NBTE was associated with composite events of mortality and stroke, we performed multivariate Cox proportional hazard regression analyses. As all patients with NBTE had metastasis and $90 \%$ of patients with NBTE had multiple territory infarc- 
tions, we constructed two different multivariate regression models to avoid multicollinearity. Model 1 included the presence of NBTE and Model 2 included metastasis and multiple territory infarctions. All statistical analyses were performed using $R$ statistical software version 3.5.1 (http://www.R-project.org). A $P<0.05$ was considered statistically significant.

\section{Results}

During the 9-year study period, 289 patients with acute ischemic stroke and active cancer were registered to the cohort. Of those, 245 patients (84.8\%) underwent echocardiography and were included in this study (123 patients underwent both TEE and TTE, 25 patients underwent TEE only, and 97 patients underwent TE only). The mean age of the included patients was $68.7 \pm 10.7$ years, and 136 patients (55.5\%) were men. Compared to patients who underwent echocardiography, those who did not undergo echocardiography were younger, had fewer atrial fibrillation and higher National Institutes of Health Stroke Scale (NIHSS) scores. Cancer types were not different between patients who did or did not undergo echocardiography (Supplementary Table 1).

\section{Comparison between the groups}

Of the 245 patients, 20 patients (8.2\%) had NBTE, 96 (39.2\%) had a cryptogenic etiology, and 129 (52.7\%) had determined etiologies. In patients with determined etiologies, those with cardioembolism were 51, those with large artery atherosclerosis were 46 , and those with two or more causes were 19 (Supplementary Table 2). Of 150 patients (61.2\%) with metastasis, NBTE was diagnosed in 20 patients (13.3\%). Compared with patients with determined etiologies, those with NBTE were younger, had more frequent multiple vascular territory involvement, lower platelet counts, lower fibrinogen levels, and higher D-dimer levels (Table 1).

\section{Metastasis, cancer type, and NBTE}

All 20 patients with NBTE had metastasis, and NBTE was not detected in patients without metastasis. The presence of metastasis was significantly higher in patients with NBTE (100\%) than in those with stroke with a cryptogenic etiology (69.8\%; $P=0.014$ after Bonferroni's correction) or those with a determined etiology (48.8\%; $P<0.001$ after Bonferroni's correction) (Table 1). The cancer type was not significantly different among the three groups (Table 1).

\section{Six-month mortality and stroke events}

All patients were followed up for 6 months after the initial stroke event. During the follow-up, 127 patients (51.8\%) developed stroke events and/or died (110 [44.9\%] died and 55 [22.4\%] patients experienced stroke events). The median survival of patients with NBTE was 44.5 days, and event-free survival of composite outcomes of mortality and stroke was 36 days. During the 6-month follow-up, 90\% (18/20) patients with NBTE died or developed stroke, while only $38.8 \%$ patients with a determined etiology died or developed stroke (50/129, $P<0.001$ ) (Table 2).

Compared to event-free patients, those with events (mortality or stroke) were younger and had lower body mass indices and higher initial NIHSS scores. They also had lower hemoglobin levels, platelet counts, and fibrinogen levels, and higher white blood cell counts and D-dimer levels (Table 3). Metastasis and multiple vascular territory involvements were more common in patients with mortality or stroke events. Cancer type was also different between patients with or without events (Table 3).

In the Kaplan-Meier survival analysis, the 6-month mortality was different among the three groups $(P<0.001)$. Patients with NBTE showed lower 6-month survival probability, lower strokefree probability, and lower event-free probability than those with cryptogenic or determined etiologies (Figure 1). Mortality, stroke events, and composite outcomes were more common in patients with a cryptogenic etiology than in those with a determined etiology (Figure 1).

\section{Causes of death}

During the follow-up, more patients with NBTE died (16/20 [80.0\%]) compared to those with a cryptogenic etiology (52/96 [54.2\%]) and a determined etiology (32/129 [32.6\%]) $(P<0.001)$. Causes of death were cancer-related in $83(75.5 \%)$, stroke-related in 21 (19.1\%), and other/unknown in six (5.5\%) patients. The cause of death did not significantly differ among the three groups $(P=0.307)$ (Table 2$)$.

\section{Stroke events}

During the 6-month follow-up, stroke events occurred in 55/245 $(22.4 \%)$ of patients; $53(21.6 \%)$ with ischemic stroke and two $(0.8 \%)$ with ICH. Stroke was diagnosed using diffusion-weighted imaging in 49 patients (89.1\%) and by CT in five patients (9.1\%). The type of imaging was uncertain in one patient who was diagnosed at another hospital (1.8\%). Stroke events occurred most frequently in patients with NBTE $(10 / 20,50 \%)$, followed by those with a cryptogenic etiology $(24 / 96,25.0 \%)$ and those with a determined etiology $(21 / 129,16.3 \%)$ (Table 2$)$. Mortality was more frequent in patients with stroke events than in those without (69.1\% [38/55] vs. $37.9 \%$ [72/190], $P<0.001)$. 
Table 1. Comparison of baseline characteristics according to stroke etiology

\begin{tabular}{|c|c|c|c|c|}
\hline Characteristic & NBTE $(n=20)$ & Cryptogenic etiology ( $n=96)$ & Determined etiology $(n=129)$ & $P$ \\
\hline \multicolumn{5}{|l|}{ Demographics } \\
\hline Age (yr) & $61.5 \pm 8.7$ & $68.1 \pm 10.1$ & $70.3 \pm 10.9$ & $0.002^{*,+}$ \\
\hline Male sex & $9(45.0)$ & $43(44.8)$ & $84(65.1)$ & $0.006^{\ddagger}$ \\
\hline \multicolumn{5}{|l|}{ Risk factors } \\
\hline Hypertension & $10(50.0)$ & $57(59.4)$ & $92(71.3)$ & 0.062 \\
\hline Diabetes mellitus & $5(25.0)$ & $25(26.0)$ & $47(36.4)$ & 0.204 \\
\hline Dyslipidemia & $5(25.0)$ & $13(13.5)$ & $28(21.7)$ & 0.228 \\
\hline Atrial fibrillation & $5(25.0)$ & $0(0)$ & $46(35.7)$ & $<0.001^{*,+}$ \\
\hline Current smoker & $1(5.0)$ & $12(12.5)$ & $17(13.2)$ & 0.581 \\
\hline Patent foramen ovale & $3(15.0)$ & $21(21.9)$ & $26(20.2)$ & 0.782 \\
\hline Body mass index $\left(\mathrm{kg} / \mathrm{m}^{2}\right)$ & $22.3 \pm 2.9$ & $22.8 \pm 3.5$ & $22.5 \pm 3.6$ & 0.676 \\
\hline \multicolumn{5}{|l|}{ Medications prior to admission } \\
\hline Antiplatelets & $3(15.0)$ & 19 (19.8) & $39(30.2)$ & 0.129 \\
\hline Anticoagulants & $3(15.0)$ & $4(4.2)$ & $15(11.6)$ & 0.066 \\
\hline Statin & $7(35.0)$ & 18 (18.8) & $37(28.7)$ & 0.138 \\
\hline Medications mainly used after admission & & & & $0.048^{\ddagger}$ \\
\hline Antiplatelets & $6(30.0)$ & $56(58.3)$ & $73(56.6)$ & \\
\hline Anticoagulants & $12(60.0)$ & $31(32.3)$ & 49 (38.0) & \\
\hline Both antiplatelets and anticoagulants & $1(5.0)$ & $2(2.1)$ & $5(3.9)$ & \\
\hline No medication & $1(5.0)$ & $7(7.3)$ & $2(1.6)$ & \\
\hline Initial NIHSS score & $7(3-12)$ & $5(2-10)$ & $5(1-12)$ & 0.547 \\
\hline Reperfusion therapy & $6(30.0)$ & $12(12.6)$ & $28(21.7)$ & 0.095 \\
\hline Multiple territory involvement & 18 (90.0) & $62(64.6)$ & $47(36.4)$ & $<0.001^{1, *}$ \\
\hline \multicolumn{5}{|l|}{ Laboratory findings } \\
\hline Hemoglobin (g/dL) & $11.2 \pm 1.6$ & $10.8 \pm 2.0$ & $11.7 \pm 2.8$ & $0.036^{\ddagger}$ \\
\hline White blood cells $\left(\times 10^{9} / \mathrm{L}\right)$ & $10.4 \pm 5.1$ & $7.8 \pm 3.9$ & $8.1 \pm 4.2$ & $0.041^{*}$ \\
\hline Platelets $\left(\times 10^{9} / \mathrm{L}\right)$ & $133 \pm 82$ & $201 \pm 113$ & $237 \pm 118$ & $<0.001^{*,+}$ \\
\hline D-dimer (mg/L) & $7.4 \pm 6.5$ & $4.8 \pm 9.0$ & $1.9 \pm 2.8$ & $<0.001^{1, \neq}$ \\
\hline Fibrinogen $(\mathrm{g} / \mathrm{L})$ & $2.6 \pm 1.6$ & $3.3 \pm 1.4$ & $3.5 \pm 1.3$ & $0.013^{+}$ \\
\hline Metastatic cancer & $20(100.0)$ & $67(69.8)$ & $63(48.8)$ & $<0.001^{*,+, *}$ \\
\hline Cancer type & & & & 0.118 \\
\hline Colorectal & $2(10.0)$ & $11(11.5)$ & $19(14.7)$ & \\
\hline Gastric/esophageal & $3(15.0)$ & $14(14.6)$ & $27(20.9)$ & \\
\hline Hepatobiliary & $6(30.0)$ & $20(20.8)$ & $12(9.3)$ & \\
\hline Pancreas & $4(20.0)$ & $9(9.4)$ & $9(7.0)$ & \\
\hline Lung & $1(5.0)$ & $21(21.9)$ & 24 (18.6) & \\
\hline Bladder/urinary tract & $2(10.0)$ & $3(3.1)$ & $6(4.7)$ & \\
\hline Female genital organ & $1(5.0)$ & $5(5.2)$ & $6(4.7)$ & \\
\hline Others & $1(5.0)$ & $13(13.5)$ & $26(20.2)$ & \\
\hline
\end{tabular}

Values are presented as mean \pm standard deviation, number (\%), or median (interquartile range).

NBTE, nonbacterial thrombotic endocarditis; NIHSS, National Institutes of Health Stroke Scale.

${ }^{*}$ NBTE vs. Cryptogenic, $P<0.05 ;{ }^{+}$NBTE vs. Determined, $P<0.05 ;{ }^{*}$ Cryptogenic vs. Determined, $P<0.05$. 
Table 2. Causes of death and type of stroke event according to stroke etiology

\begin{tabular}{|c|c|c|c|c|}
\hline Variable & NBTE $(n=20)$ & Cryptogenic etiology $(n=96)$ & Determined etiology $(n=129)$ & $P$ \\
\hline Composite outcomes of mortality and stroke & $18(90)$ & $59(61.5)$ & $50(38.8)$ & $<0.001^{*, t, *}$ \\
\hline Mortality & $16(80)$ & $52(54.2)$ & $32(32.6)$ & $<0.001^{t, \neq}$ \\
\hline Causes of death & & & & 0.307 \\
\hline Cancer-related & $13(81.2)$ & $43(82.7)$ & $27(64.3)$ & \\
\hline Stroke-related & $2(12.5)$ & $7(13.5)$ & $12(28.6)$ & \\
\hline Other/unknown cause & $1(6.3)$ & $2(3.8)$ & $3(7.1)$ & \\
\hline Any stroke events & $10(50)$ & $24(25.0)$ & $21(16.3)$ & $<0.003^{+}$ \\
\hline \multicolumn{5}{|l|}{ Stroke type } \\
\hline Cerebral infarction & $10(100)$ & 23 (95.8) & $20(95.2)$ & \\
\hline Intracerebral hemorrhage & $0(0)$ & $1(4.2)$ & $1(4.8)$ & \\
\hline
\end{tabular}

Values are presented as number (\%).

NBTE, nonbacterial thrombotic endocarditis.

${ }^{*}$ NBTE vs. Cryptogenic, $P<0.05 ;{ }^{+}$NBTE vs. Determined, $P<0.05 ;{ }^{*}$ Cryptogenic vs. Determined, $P<0.05$.

\section{Factors associated with mortality and stroke events}

In the multivariate Cox proportional hazard analysis, female sex and a lower body mass index were associated with composite outcomes of mortality and stroke both in Model 1 and Model 2. The composite outcomes were independently associated with the presence of NBTE (hazard ratio [HR], 1.941; 95\% confidence interval [CI], 1.052 to 3.690) (Table 4, Model 1). The presence of metastasis ( $\mathrm{HR}, 2.870 ; 95 \% \mathrm{Cl}, 1.648$ to 4.996$)$ and the involvement of multiple vascular territories $(H R, 2.524$; $95 \% \mathrm{Cl}, 1.570$ to 4.058 ) were also independently associated with the composite outcomes (Table 4, Model 2).

\section{Discussion}

In this study, NBTE was diagnosed in approximately one of 11 stroke patients with active cancer. NBTE was also found exclusively in patients with metastasis. In addition, stroke patients with NBTE had a very high risk of mortality and stroke events during a 6-month follow-up.

The pathomechanism of NBTE remains unknown. Autopsy studies in patients with NBTE have shown that cardiac vegetation is mainly composed of platelets, ${ }^{21}$ and examinations of thrombi retrieved during mechanical thrombectomy in stroke patients with NBTE have indicated very high platelet and low erythrocyte fractions. ${ }^{11,22}$ These studies suggest that plateletmediated mechanisms play a key role in the development of cancer-associated NBTE. The role of platelets in cancer has been extensively studied; platelets play a crucial role in tumor growth and metastasis. ${ }^{23}$

Our study demonstrated that NBTE occurred exclusively in patients with metastasis. Platelets can contribute to tumor in- vasion and metastasis via several mechanisms. Platelets are essential for the survival of circulating tumor cells, ${ }^{24}$ and these cells rapidly associate with platelets via their receptors and induce tumor cell-induced platelet aggregation (TCIPA). ${ }^{25}$ This TCIPA is critical for tumor cell survival as platelets form a physical shield around tumor cells, protecting them from recognition and lysis by natural killer cells and shear-induced damage. ${ }^{26,27}$ These findings suggest that the association of tumor cells with platelets during metastasis may somehow contribute to the development of platelet-rich vegetation in NBTE.

Stroke patients with active cancer have a substantial risk of recurrent stroke. In a retrospective study, ischemic stroke recurred in 16\% patients within 6 months of the initial stroke. ${ }^{15}$ In the present study cohort, ischemic stroke recurred in 21.6\% patients and ICH developed in 0.8\% patients. Particularly, 50\% patients with NBTE developed stroke within 6 months, significantly higher than those without NBTE. Ninety percent of patients with NBTE had lesions involving multiple vascular territories, which suggests multiple embolization from the heart. Patients with NBTE may be prone to a high risk of multiple thromboembolism because the platelet-rich vegetation (thrombus) is attached to the fast-moving cardiac valves.

This study showed that mortality differed significantly among the three etiology categories of stroke patients with active cancer. During the 6 months of follow-up, 80\% patients with NBTE died. However, less than one-third of the patients with a determined etiology died. Patients with a cryptogenic etiology had an intermediate risk of 6-month mortality. The high mortality in patients with NBTE may be associated with metastasis since all patients with NBTE had it. In our previous study, 67.1\% stroke patients with metastatic cancer died within 6 months, ${ }^{20}$ but the 6-month mortality in patients with 
Table 3. Comparison of characteristics between patients with and without mortality/stroke events

\begin{tabular}{|c|c|c|c|}
\hline Characteristic & $\begin{array}{c}\text { No events } \\
(n=118)\end{array}$ & $\begin{array}{l}\text { Events } \\
(n=127)\end{array}$ & $P$ \\
\hline \multicolumn{4}{|l|}{ Demographics } \\
\hline Age $(y r)$ & $68.4 \pm 9.9$ & $69.0 \pm 11.4$ & 0.664 \\
\hline Male sex & $80(67.8)$ & $56(44.1)$ & $<0.001$ \\
\hline \multicolumn{4}{|l|}{ Risk factors } \\
\hline Hypertension & 79 (66.9) & $80(63.0)$ & 0.607 \\
\hline Diabetes mellitus & 39 (33.1) & 38 (29.9) & 0.697 \\
\hline Dyslipidemia & 27 (22.9) & 19 (15.0) & 0.155 \\
\hline Atrial fibrillation & $26(22.0)$ & 25 (19.7) & 0.768 \\
\hline Current smoker & 20 (16.9) & $10(7.9)$ & 0.049 \\
\hline Body mass index $\left(\mathrm{kg} / \mathrm{m}^{2}\right)$ & $23.2 \pm 3.2$ & $22.0 \pm 3.8$ & 0.010 \\
\hline Metastatic cancer & $42(35.6)$ & $108(85.0)$ & $<0.001$ \\
\hline Cancer type & & & 0.020 \\
\hline Colorectal & $19(16.1)$ & $13(10.2)$ & \\
\hline Gastric/esophageal & $22(18.6)$ & $22(17.3)$ & \\
\hline Hepatobiliary & $13(11.0)$ & $25(19.7)$ & \\
\hline Pancreas & $5(4.2)$ & $17(13.4)$ & \\
\hline Lung & $21(17.8)$ & 25 (19.7) & \\
\hline Bladder/urinary tract & $8(6.8)$ & $3(2.4)$ & \\
\hline Female genital organ & $5(4.2)$ & $7(5.5)$ & \\
\hline Others & $25(21.2)$ & $15(11.8)$ & \\
\hline Initial NIHSS score & $3(1-10)$ & $6(2-11.5)$ & 0.007 \\
\hline Reperfusion therapy & $25(21.2)$ & $21(16.7)$ & 0.460 \\
\hline Multiple vascular territory involvement & $36(30.5)$ & $91(71.7)$ & $<0.001$ \\
\hline Stroke etiology & & & $<0.001$ \\
\hline NBTE & $2(10.0)$ & $18(90.0)$ & \\
\hline Cryptogenic & $37(38.5)$ & $59(61.5)$ & \\
\hline Determined & $79(61.2)$ & $50(38.8)$ & \\
\hline \multicolumn{4}{|l|}{ Laboratory findings } \\
\hline Hemoglobin (g/dL) & $11.6 \pm 2.1$ & $11.0 \pm 2.7$ & 0.033 \\
\hline White blood cells $\left(\times 10^{9} / \mathrm{L}\right)$ & $7.5 \pm 3.7$ & $8.9 \pm 4.5$ & 0.008 \\
\hline Platelets $\left(\times 10^{9} / L\right)$ & $243 \pm 112$ & $188 \pm 116$ & $<0.001$ \\
\hline D-dimer (mg/L) & $1.1 \pm 1.5$ & $5.7 \pm 8.3$ & $<0.001$ \\
\hline Fibrinogen $(\mathrm{g} / \mathrm{L})$ & $3.6 \pm 1.2$ & $3.2 \pm 1.5$ & 0.021 \\
\hline
\end{tabular}

Values are presented as mean \pm standard deviation, number (\%), or median (interquartile range).

NIHSS, National Institutes of Health Stroke Scale; NBTE, nonbacterial thrombotic endocarditis.

NBTE was even higher. In the present study, mortality was also higher in patients who developed recurrent stroke; therefore, frequent stroke reoccurrence in patients with NBTE might also increase the risk of death.

In this study, patients with NBTE had higher D-dimer levels and more frequent multiple vascular territory involvement than
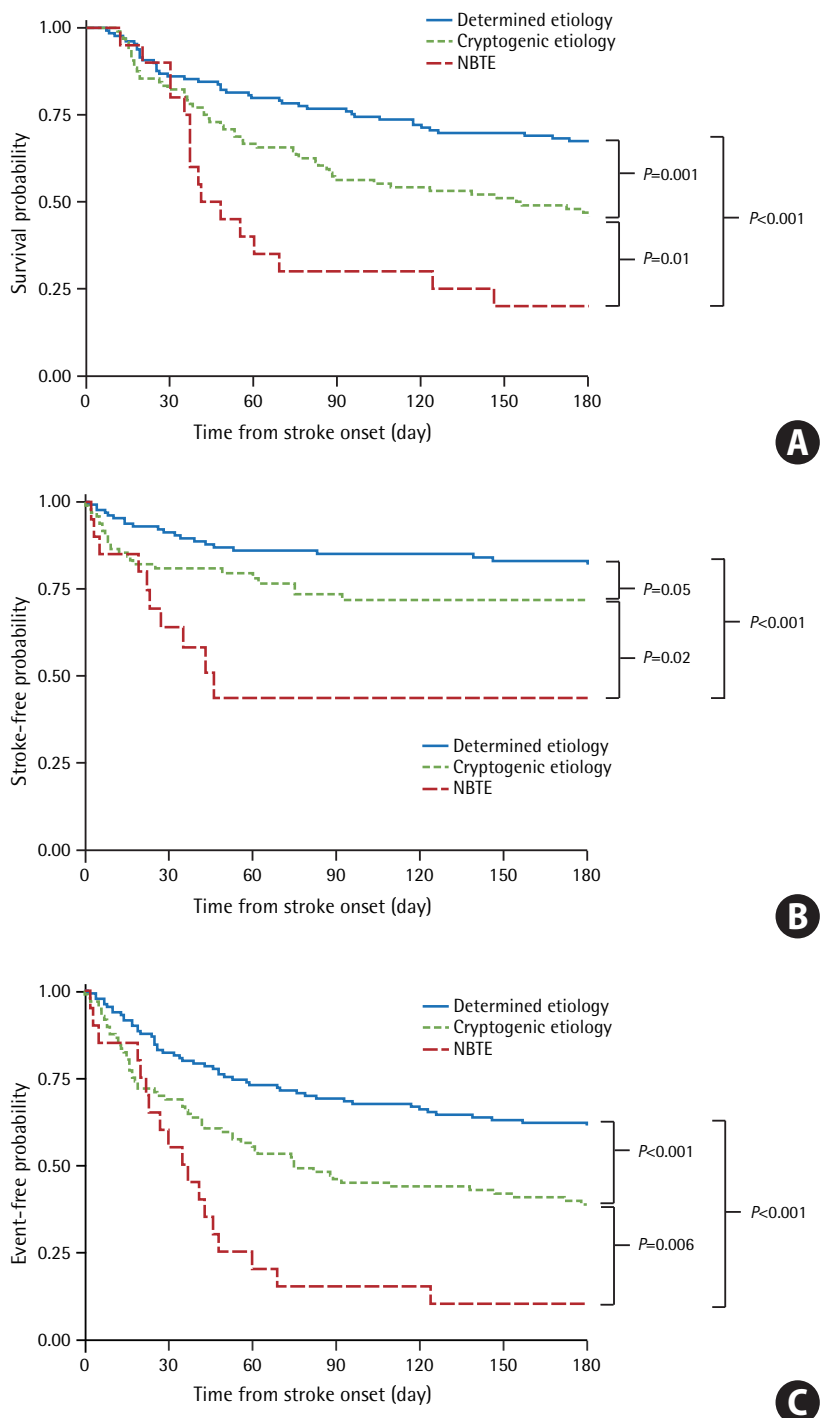

Figure 1. Kaplan-Meyer analysis of (A) mortality, (B) stroke events, and (C) composite outcomes of mortality and stroke events during the 6-month follow-up. Bonferroni's correction was performed for intergroup comparisons. NBTE, nonbacterial thrombotic endocarditis.

those of patients with stroke of a determined etiology. These are known characteristics of cancer-related stroke. ${ }^{3}$ Our findings suggest that some features of cancer-related stroke may be associated with NBTE. ${ }^{28,29}$ Patients with a cryptogenic etiology had intermediate characteristics between those with NBTE and those with a determined etiology. Although NBTE is often not diagnosed despite TEE being performed ${ }^{30}$ NBTE has been suggested as the likely cause of cryptogenic stroke in many patients with cancer. ${ }^{29}$ Therefore, undiagnosed NBTE may have been present in some patients with a cryptogenic etiology.

This study has several limitations. First, although patients were enrolled prospectively, analysis for this study was per- 
Table 4. Cox models for 6-month composite outcomes of mortality/stroke events

\begin{tabular}{|c|c|c|c|c|c|c|}
\hline \multirow{3}{*}{ Variable } & \multirow{2}{*}{\multicolumn{2}{|c|}{ Univariate analysis }} & \multicolumn{4}{|c|}{ Multivariate analysis } \\
\hline & & & \multicolumn{2}{|l|}{ Model 1} & \multicolumn{2}{|l|}{ Model 2} \\
\hline & $\mathrm{HR}(95 \% \mathrm{Cl})$ & $P$ & HR $(95 \% \mathrm{Cl})$ & $P$ & $\mathrm{HR}(95 \% \mathrm{Cl})$ & $P$ \\
\hline \multicolumn{7}{|l|}{ Demographics } \\
\hline Age (yr) & 0.999 (0.982-1.017) & 0.931 & 1.015 (0.996-1.034) & 0.137 & 1.011 (0.992-1.030) & 0.269 \\
\hline Male sex & $0.471(0.331-0.669)$ & $<0.001$ & $0.540(0.347-0.851)$ & 0.007 & $0.520(0.333-0.810)$ & 0.004 \\
\hline \multicolumn{7}{|l|}{ Risk factors } \\
\hline Hypertension & $0.841(0.587-1.206)$ & 0.347 & & & & \\
\hline Diabetes mellitus & $0.877(0.600-1.282)$ & 0.498 & & & & \\
\hline Dyslipidemia & 0.665 (0.408-1.083) & 0.101 & 0.878 (0.519-1.463) & 0.625 & $0.948(0.561-1.601)$ & 0.840 \\
\hline Atrial fibrillation & $0.902(0.582-1.396)$ & 0.643 & & & & \\
\hline Current smoker & $0.500(0.262-0.955)$ & 0.036 & $0.938(0.429-1.807)$ & 0.862 & $0.856(0.418-1.753)$ & 0.670 \\
\hline Body mass index $\left(\mathrm{kg} / \mathrm{m}^{2}\right)$ & $0.927(0.878-0.979)$ & 0.007 & $0.926(0.869-0.980)$ & 0.012 & $0.929(0.875-0.986)$ & 0.015 \\
\hline \multicolumn{7}{|l|}{ Stroke etiology } \\
\hline Determined & Reference & & Reference & & & \\
\hline Cryptogenic & $1.976(1.355-2.882)$ & $<0.001$ & 1.447 (0.938-2.218) & 0.090 & & \\
\hline NBTE & 4.099 (2.371-7.086) & $<0.001$ & 1.941 (1.052-3.690) & 0.038 & & \\
\hline \multicolumn{7}{|l|}{ Medications mainly used after admission* } \\
\hline Antiplatelets & Reference & & Reference & & Reference & \\
\hline Anticoagulants & $1.810(1.277-2.567)$ & 0.001 & 1.353 (0.915-1.999) & 0.130 & $0.958(0.644-1.427)$ & 0.834 \\
\hline Presence of metastasis & $5.332(3.264-8.708)$ & $<0.001$ & & & $2.870(1.648-4.996)$ & $<0.001$ \\
\hline \multicolumn{7}{|l|}{ Cancer type } \\
\hline Colorectal & $1.113(0.530-2.339)$ & 0.778 & $0.694(0.326-1.565)$ & 0.362 & $0.796(0.360-1.761)$ & 0.573 \\
\hline Gastric/esophageal & 1.521 (0.789-2.932) & 0.210 & 1.415 (0.675-2.839) & 0.342 & 1.399 (0.682-2.868) & 0.359 \\
\hline Hepatobiliary & $2.196(1.157-4.167)$ & 0.016 & $1.522(0.745-2.997)$ & 0.236 & $1.312(0.649-2.656)$ & 0.450 \\
\hline Pancreas & $2.812(1.402-5.638)$ & 0.004 & $1.093(0.513-2.460)$ & 0.826 & $0.767(0.339-1.734)$ & 0.523 \\
\hline Lung & $1.628(0.858-3.087)$ & 0.136 & $1.330(0.670-2.568)$ & 0.404 & $1.084(0.550-2.133)$ & 0.816 \\
\hline Bladder/urinary tract & $0.648(0.188-2.238)$ & 0.493 & $0.686(0.201-2.788)$ & 0.576 & $1.120(0.293-4.280)$ & 0.869 \\
\hline Female genital organ & $2.059(0.839-5.052)$ & 0.115 & 1.180 (0.477-3.289) & 0.738 & $1.115(0.424-2.936)$ & 0.825 \\
\hline Others & Reference & & Reference & & Reference & \\
\hline Initial NIHSS score & $1.031(1.004-1.058)$ & 0.024 & $1.023(0.994-1.056)$ & 0.140 & $1.028(0.998-1.060)$ & 0.071 \\
\hline Reperfusion therapy & $0.808(0.506-1.291)$ & 0.372 & & & & \\
\hline Multiple vascular territory involvement & $3.662(2.482-5.403)$ & $<0.001$ & & & $2.524(1.570-4.058)$ & $<0.001$ \\
\hline \multicolumn{7}{|l|}{ Laboratory findings } \\
\hline Hemoglobin (g/dL) & $0.918(0.847-0.994)$ & 0.034 & $0.960(0.864-1.046)$ & 0.415 & $1.007(0.920-1.102)$ & 0.879 \\
\hline White blood cells $\left(\times 10^{9} / \mathrm{L}\right)$ & $1.061(1.020-1.104)$ & 0.004 & $1.095(1.040-1.145)$ & $<0.001$ & $1.094(1.040-1.151)$ & 0.001 \\
\hline Platelets $\left(\times 10^{9} / L\right)$ & $0.996(0.994-0.998)$ & $<0.001$ & 0.997 (0.995-0.999) & 0.003 & $0.997(0.996-0.999)$ & 0.006 \\
\hline D-dimer (mg/L) & $1.078(1.061-1.095)$ & $<0.001$ & $1.053(1.028-1.078)$ & $<0.001$ & $1.033(1.006-1.062)$ & 0.018 \\
\hline Fibrinogen $(g / L)$ & $0.998(0.996-0.999)$ & 0.002 & 0.999 (0.998-1.001) & 0.480 & 0.999 (0.997-1.001) & 0.174 \\
\hline
\end{tabular}

$\mathrm{HR}$, hazard ratio; $\mathrm{Cl}$, confidence interval; NBTE, nonbacterial thrombotic endocarditis; NIHSS, National Institutes of Health Stroke Scale.

*Excluded eight patients with both antiplatelet and anticoagulant, 10 patients without antithrombotics.

formed retrospectively at a single center. In addition, as the study hospital has a dedicated cancer center, the frequency of cancer, particularly that of advanced cancer or metastasis, might be higher than that in other more general hospitals. Second, although most patients (85\%) with active cancer underwent echocardiography, TEE (the gold standard for diagnosis 
of vegetation ${ }^{30,31}$ ) was used in only $60.4 \%$. Although TEE is one of the routine evaluations in our protocol, it was impractical to perform TEE in all patients (it is semi-invasive and requires patient cooperation and informed consent). Vegetation is often invisible on $T E$, and even when using $T E E$, a small vegetation may not be detected. Therefore, the presence of NBTE in this study might have been underestimated. Third, the antithrombotic treatment for stroke prevention in our study population was not controlled. Therefore, the influence of preventive treatment on stroke recurrence is unknown. Finally, our explanation on the role of platelets in metastasis and development of NBTE is speculative.

\section{Conclusions}

NBTE should be suspected as a potential mechanism of stroke in patients with metastatic cancer, particularly in those without determinable etiologies. We also showed that patients with NBTE have a very high short-term risk of recurrent stroke and mortality. In addition, mortality was higher in patients who developed recurrent stroke. In this regard, efforts are necessary to reduce the risk of stroke recurrence in these patients. Further studies are necessary to determine optimal preventive treatment in stroke patients with cancer-associated NBTE.

\section{Supplementary materials}

Supplementary materials related to this article can be found online at https://doi.org/10.5853/jos.2020.00619.

\section{Disclosure}

The authors have no financial conflicts of interest.

\section{Acknowledgments}

This study was supported by the Basic Science Research Program through the National Research Foundation of Korea (NRF) funded by the Ministry of Education (NRF-2018R1A2A3074996), the National Research Foundation of Korea grant funded by the Korean government (NRF-2017R1C1B5076990), and a faculty research grant of Yonsei University College of Medicine (62018-0185).

\section{References}

1. Navi BB, Reiner AS, Kamel $H$, ladecola C, Okin PM, Elkind MSV, et al. Risk of arterial thromboembolism in patients with can- cer. J Am Coll Cardiol 2017;70:926-938.

2. Kim K, Kim J, Ahn SH, Ha WS, Koo YJ, Kim DJ, et al. Histopathological findings of intracranial thrombi in nonbacterial thrombotic endocarditis. J Stroke 2017;19:367-369.

3. Navi BB, ladecola $C$. Ischemic stroke in cancer patients: a review of an underappreciated pathology. Ann Neurol 2018;83:873883.

4. Bang OY, Chung JW, Lee MJ, Seo WK, Kim GM, Ahn MJ, et al. Cancer-related stroke: an emerging subtype of ischemic stroke with unique pathomechanisms. J Stroke 2020;22:1-10.

5. Reagan TJ, Okazaki $\mathrm{H}$. The thrombotic syndrome associated with carcinoma. A clinical and neuropathologic study. Arch Neurol 1974;31:390-395.

6. Chen L, Li Y, Gebre W, Lin JH. Myocardial and cerebral infarction due to nonbacterial thrombotic endocarditis as an initial presentation of pancreatic adenocarcinoma. Arch Pathol Lab Med 2004;128:1307-1308.

7. Yamane $A$, Sadahiro $H$, Goto $H$, Inamura $A$, Ishihara $H, O$ ka $F$, et al. Multiple ischemic strokes caused by nonbacterial thrombotic endocarditis because of gallbladder cancer: a case report. J Stroke Cerebrovasc Dis 2014;23:1727-1729.

8. Bonnerot M, Humbertjean L, Mione G, Lacour JC, Derelle AL, Sanchez JC, et al. Cerebral ischemic events in patients with pancreatic cancer: a retrospective cohort study of 17 patients and a literature review. Medicine (Baltimore) 2016;95:e4009.

9. Navi BB, Howard G, Howard VJ, Zhao H, Judd SE, Elkind MSV, et al. New diagnosis of cancer and the risk of subsequent cerebrovascular events. Neurology 2018;90:e2025-e2033.

10. Rogers LR, Cho ES, Kempin S, Posner JB. Cerebral infarction from non-bacterial thrombotic endocarditis. Clinical and pathological study including the effects of anticoagulation. Am J Med 1987;83:746-756.

11. Park H, Kim J, Ha J, Hwang IG, Song TJ, Yoo J, et al. Histological features of intracranial thrombi in stroke patients with cancer. Ann Neurol 2019;86:143-149.

12. Gay $\sqcup$, Felding-Habermann B. Contribution of platelets to tumour metastasis. Nat Rev Cancer 2011;11:123-134.

13. Deppisch LM, Fayemi AO. Non-bacterial thrombotic endocarditis: clinicopathologic correlations. Am Heart J 1976;92:723729.

14. Taccone FS, Jeangette SM, Blecic SA. First-ever stroke as initial presentation of systemic cancer. J Stroke Cerebrovasc Dis 2008;17:169-174.

15. Navi BB, Singer $S$, Merkler AE, Cheng NT, Stone JB, Kamel $H$, et al. Cryptogenic subtype predicts reduced survival among cancer patients with ischemic stroke. Stroke 2014;45:22922297.

16. Erdur $H$, Scheitz JF, Ebinger $M$, Rocco $A$, Grittner $U$, Meisel $A$, 
et al. In-hospital stroke recurrence and stroke after transient ischemic attack: frequency and risk factors. Stroke 2015;46: 1031-1037.

17. Cho HJ, Choi HY, Kim YD, Nam HS, Han SW, Ha JW, et al. Transoesophageal echocardiography in patients with acute stroke with sinus rhythm and no cardiac disease history. $J$ Neurol Neurosurg Psychiatry 2010;81:412-415.

18. Lee AY, Levine MN, Baker RI, Bowden C, Kakkar AK, Prins M, et al. Low-molecular-weight heparin versus a coumarin for the prevention of recurrent venous thromboembolism in patients with cancer. N Engl J Med 2003;349:146-153.

19. Yoo J, Song D, Baek JH, Kim K, Kim J, Song TJ, et al. Poor longterm outcomes in stroke patients with asymptomatic coronary artery disease in heart CT. Atherosclerosis 2017;265:7-13.

20. Yoo J, Nam HS, Kim YD, Lee HS, Heo JH. Short-term outcome of ischemic stroke patients with systemic malignancy. Stroke 2019;50:507-511.

21. el-Shami K, Griffiths E, Streiff M. Nonbacterial thrombotic endocarditis in cancer patients: pathogenesis, diagnosis, and treatment. Oncologist 2007;12:518-523.

22. Heo JH, Nam HS, Kim YD, Choi JK, Kim BM, Kim DJ, et al. Pathophysiologic and therapeutic perspectives based on thrombus histology in stroke. J Stroke 2020;22:64-75.

23. Schlesinger $M$. Role of platelets and platelet receptors in cancer metastasis. J Hematol Oncol 2018;11:125.

24. Xu XR, Yousef GM, Ni H. Cancer and platelet crosstalk: op- portunities and challenges for aspirin and other antiplatelet agents. Blood 2018;131:1777-1789.

25. Jurasz P, Alonso-Escolano D, Radomski MW. Platelet: cancer interactions. Mechanisms and pharmacology of tumour cellinduced platelet aggregation. Br J Pharmacol 2004;143:819826.

26. Nieswandt $B$, Hafner M, Echtenacher B, Mannel DN. Lysis of tumor cells by natural killer cells in mice is impeded by platelets. Cancer Res 1999;59:1295-1300.

27. Egan K, Cooke N, Kenny D. Living in shear: platelets protect cancer cells from shear induced damage. Clin Exp Metastasis 2014;31:697-704.

28. Bal S, Menon B, Demchuk A. Relationship of endocarditis, disseminated intravascular coagulation, and embolic signals in cancer with stroke. Ann Neurol 2012;71:146.

29. Navi BB, Singer $S$, Merkler AE, Cheng NT, Stone JB, Kamel $H$, et al. Recurrent thromboembolic events after ischemic stroke in patients with cancer. Neurology 2014;83:26-33.

30. Merkler AE, Navi BB, Singer S, Cheng NT, Stone JB, Kamel H, et al. Diagnostic yield of echocardiography in cancer patients with ischemic stroke. J Neurooncol 2015;123:115-121.

31. Dutta T, Karas MG, Segal AZ, Kizer JR. Yield of transesophageal echocardiography for nonbacterial thrombotic endocarditis and other cardiac sources of embolism in cancer patients with cerebral ischemia. Am J Cardiol 2006;97:894898. 
Supplementary Table 1. Comparison between patients with and without transesophageal echocardiography

\begin{tabular}{|c|c|c|c|}
\hline Variable & $\begin{array}{l}\text { Without echocardiography } \\
\qquad(\mathrm{n}=44)\end{array}$ & $\begin{array}{l}\text { With echocardiography } \\
\qquad(n=245)\end{array}$ & $P$ \\
\hline \multicolumn{4}{|l|}{ Demographics } \\
\hline Age (yr) & $64.1 \pm 10.5$ & $68.7 \pm 10.7$ & 0.010 \\
\hline Male sex & $25(56.8)$ & $136(55.5)$ & $>0.999$ \\
\hline \multicolumn{4}{|l|}{ Risk factors } \\
\hline Hypertension & 25 (56.8) & $159(64.9)$ & 0.392 \\
\hline Diabetes mellitus & 14 (31.8) & 77 (31.4) & $>0.999$ \\
\hline Dyslipidemia & $7(15.9)$ & 46 (18.8) & 0.810 \\
\hline Atrial fibrillation & $2(4.6)$ & $51(20.8)$ & 0.018 \\
\hline Current smoker & $7(15.9)$ & 46 (18.8) & 0.810 \\
\hline Body mass index $\left(\mathrm{kg} / \mathrm{m}^{2}\right)$ & $21.8 \pm 3.2$ & $22.6 \pm 3.5$ & 0.162 \\
\hline Metastatic cancer & $34(77.3)$ & $150(61.2)$ & 0.062 \\
\hline Cancer type & & & 0.556 \\
\hline Colorectal & $4(9.1)$ & $32(13.1)$ & \\
\hline Gastric/esophageal & $6(13.6)$ & $44(18.0)$ & \\
\hline Hepatobiliary & $4(9.1)$ & $38(15.5)$ & \\
\hline Pancreas & $8(18.2)$ & $22(9.0)$ & \\
\hline Lung & $11(25.0)$ & 46 (18.8) & \\
\hline Bladder/urinary tract & $2(4.5)$ & $11(4.5)$ & \\
\hline Female genital organ & $1(2.3)$ & $12(4.9)$ & \\
\hline Others & $8(18.2)$ & 40 (16.3) & \\
\hline Initial NIHSS score & $6.5(3-15)$ & $5(2-11)$ & 0.041 \\
\hline Reperfusion therapy & $8(18.2)$ & 46 (18.9) & $>0.999$ \\
\hline Multiple vascular territory involvement & $20(45.5)$ & $127(51.8)$ & 0.538 \\
\hline \multicolumn{4}{|l|}{ Laboratory findings } \\
\hline Hemoglobin (g/dL) & $11.1 \pm 1.8$ & $11.3 \pm 2.4$ & 0.557 \\
\hline White blood cells $\left(\times 10^{9} / \mathrm{L}\right)$ & $9.1 \pm 5.0$ & $8.2 \pm 4.2$ & 0.285 \\
\hline Platelets $\left(\times 10^{9} / \mathrm{L}\right)$ & $219 \pm 120$ & $214 \pm 117$ & 0.806 \\
\hline D-dimer (mg/L) & $5.2 \pm 8.0$ & $3.5 \pm 6.5$ & 0.201 \\
\hline Fibrinogen $(\mathrm{g} / \mathrm{L})$ & $3.3 \pm 1.4$ & $3.3 \pm 1.4$ & 0.870 \\
\hline
\end{tabular}

Values are presented as mean \pm standard deviation, number (\%), or median (interquartile range). NIHSS, National Institutes of Health Stroke Scale. 
Supplementary Table 2. Etiologic mechanisms of stroke in 129 patients with a determined etiology

\begin{tabular}{lc}
\hline Mechanism & Etiology \\
\hline Cardioembolism & 51 \\
Atrial fibrillation & 31 \\
Patent foramen ovale (with venous thrombosis) & 7 \\
Akinetic left ventricular segment & 3 \\
Hypokinetic left ventricular segment & 3 \\
Valvular heart disease (prosthetic valve replacement) & 2 \\
Cardiac thrombus & 2 \\
Atrial septal aneurysm & 1 \\
Status of inserted pacemaker (sick sinus syndrome) & 1 \\
Infective endocarditis & 1 \\
Large artery atherosclerosis & 46 \\
Small artery disease & 10 \\
Other determined etiologies & 3 \\
Two or more causes & 19 \\
Large artery atherosclerosis+cardioembolism & 14 \\
Atrial fibrillation & 11 \\
Atrial flutter & 1 \\
Prosthetic valve replacement & 2 \\
Spontaneous echo contrast & 1 \\
Large artery atherosclerosis+small artery disease & 1 \\
Cardioembolism+small artery disease & 3 \\
Atrial fibrillation & 2 \\
\hline
\end{tabular}

